\title{
Tapentadol for the management of neuropathic pain from oxaliplatin chemotherapy: A case report
}

\author{
Mugabure Bujedo Borja ${ }^{1,2}$ \\ ${ }^{1}$ Department of Anesthesiology, Critical Care and Pain Medicine, Donostia University Hospital, San Sebastian, 20014, Spain \\ ${ }^{2}$ Pain Relief Unit, Chronic Pain Management, Donostia University Hospital, San Sebastian, 20014, Spain
}

Email address:

mugabure@yahoo.es

\section{To cite this article:}

Mugabure Bujedo Borja. Tapentadol for the Management of Neuropathic Pain from Oxaliplatin Chemotherapy: A Case Report. American Journal of Internal Medicine. Special Issue: Neuropathic Pain. Vol. 2, No. 6-1, 2014, pp. 1-4. doi: 10.11648/j.ajim.s.2014020601.11

\begin{abstract}
Chronic pain is one of the most important health problems from the XXI century due to its often challenging to address appropriately. Opioids are broad-spectrum analgesics that may be beneficial to alleviate the intense perception of analgesia in patients suffering with pain. They have been one of the most controversial analgesics, in part because of their potential for addiction. Although patients with severe chronic pain may respond to treatment with an opioid analgesic, opioids are often associated with adverse effects that may lead patients to disrupt or discontinue therapy. In addition, opioid drugs alone may not be effective for all types of chronic pain, including neuropathic pain (NP). Opioids or any currently available analgesic will not provide effective analgesia for every patient with chronic NP, but overall, opioids are considered to be a second or third line class of analgesics that may provide reasonable analgesia to some patients with chronic NP. Although opioids may alleviate chronic pain, overall, NP tends to be less opioid responsive than nociceptive pain. Tapentadol prolonged release (PR), a centrally acting analgesic with 2 mechanisms of action, $\mu$-opioid receptor agonism (MOR) and noradrenaline reuptake inhibition (NRI), provides strong and reliable analgesia across a range of indications, including nociceptive, neuropathic, and mixed types of chronic pain, and is associated with an improved tolerability profile relative to classic opioid analgesics. The purpose of this article was to demonstrate clinical efficacy of tapentadol PR in association to pregabalin for the treatment of neuropathic pain from oxaliplatin chemotherapy.
\end{abstract}

Keywords: Chronic Pain, Neuropathic Pain, Opioids, Tapentadol, Oxaliplatin

\section{Introduction}

The International Association for the Study of Pain (IASP) defines neuropathic pain (NP) as a triggered pain caused by injury or primary dysfunction from either central or peripheral nervous system. The epidemiology of NP has not been adequately studied but is estimated to affect $1-3 \%$ of the population. The origin of NP may be so different such as infection, trauma, metabolic disorders, chemotherapy, surgery and/or neural compression. Patients usually report a continuous burning pain, which may be accompanied by paroxysms of lancinating or electrical pain; this one may be spontaneous or secondary to small stimuli. Pain may occur as a heightened response to a painful stimulus (hyperalgesia), or in response to non-painful stimuli (allodynia). All these symptoms are linked to different mechanisms and therefore susceptible to a wide variability in their therapeutic approach. In this field, the opioid drugs have generated so much controversy in relation to its poor balance between tolerability and efficacy. As a result, NP continues to be one of the greatest health challenges of the twenty-first century that remains unresolved satisfactorily due to its difficulty in both diagnosis and treatment. This fact increases either patient's dissatisfaction or Health Systems' cost [1].

\section{Clinic Case Description}

The author reports a case of severe peripheral polyneuropathy secondary to oxaliplatin chemotherapy with great symptomatic relief after multimodal medical treatment with antidepressant, anticonvulsant and tapentadol.

\subsection{Clinical Diagnosis}

\subsubsection{Medical History}

We present a 59-year-old male diagnosed with invasive colon adenocarcinoma stage pT3. He had been surgically 
operated on performing a resection from left hemi colectomy and also preoperative radiotherapy. After surgery, he received chemotherapy (CT) (5-FLU, oxaliplatin and FOLFOX-cycles). After several CT sessions, six months later, the patient presented NP in hands and feet, with continuous dysesthesias and lancinating crisis, especially during the night. (Visual Analogue Scale: VAS 7 at day and 9 at night).

\subsubsection{Physical Examination}

Physical examination revealed allodynia in either hands or feet to both thermal and tactile stimuli, tenderness in the skin prick test and dysesthesia. No alterations of motor function were detected.

\subsubsection{Diagnoses Evaluations}

The questionnaire items Douleur Neuropathique-4 (DN4) consists of 10 items. Descriptions and signs of pain are evaluated with 1 (yes) or 0 (no) to identify patients who have likely to have a large component of NP. The scores of the individual items are summed to obtain a maximum total score of 10 with a minimum point $\geq 4$. The diagnosis of NP was confirmed in our patient with the test DN-4 (8/10). Other tests such as nuclear magnetic resonance (NMR) and electromyogram (EMG) dismissed compressive pathology of cervical and lumbar spinal canal and acute radiculopathy.

\subsubsection{Final Exclusion Diagnosis}

Based on clinical history and physical examination a distal sensory polyneuropathy secondary to chemotherapy was diagnosed.

\subsection{Treatment and Clinical Evolution}

The Neurologist medical doctor initiated oral gabapentin $900 \mathrm{mg} /$ day and amitriptyline $25 \mathrm{mg}$ single dose by night; however it was overall not well tolerated by daytime due to drowsiness and blurred vision. The physician switched to duloxetine $60 \mathrm{mg}$ /day and pregabalin $150 \mathrm{mg} / 12 \mathrm{~h}$. Daytime pain crisis were improved but not nocturnal ones, which depressed and anguished him, so trazodone hydrochloride 100 $\mathrm{mg}$ at night was added to the prescription, duloxetine was removed and the patient was sent to the Chronic Pain Unit.

\subsection{1. $1^{\text {st }}$ Medical Visit}

EVA during the day was estimated as 5 and the stabbing pain that woke the patient up at night was 8 . A propose to increase pregabalin night dose to $300 \mathrm{mg}$ was made and Paracetamol $325 \mathrm{mg}+37.5 \mathrm{mg}$ tramadol was also added in progressive doses (1-2 pills/ $8 \mathrm{~h}$ ) with a daily laxative (lactulose).

\subsection{2. $2^{\text {nd }}$ Medical Visit One Month Later}

The new treatment was not well tolerated by either nausea or constipation and therefore the patient had removed tramadol. Slight improvement in night sleeping was noted during this period. The medical team decided to add tapentadol on rising doses as follows; tapentadol $50 \mathrm{mg}$ night $1 \mathrm{st}$ week, $50 \mathrm{mg} / 12 \mathrm{~h}$ 2nd week, $50 \mathrm{mg}$ morning and $100 \mathrm{mg}$ night $3 \mathrm{rd}$ week and $100 \mathrm{mg} / 12 \mathrm{~h}$ 4th week.

\subsection{3. $3^{\text {th }}$ Medical Visit One Month Later}

A great improvement in either night or daytime pain was detected with tapentadol $200 \mathrm{mg} /$ day total dose. EVA was estimated to be $<3$ during the night and $<5$ during the day. The overall tapentadol dose was increased to $150 \mathrm{mg} / 12 \mathrm{~h}$.

\subsection{4. $4^{\text {th }}$ Medical Visit Three Months Later}

General wellbeing was improving with less number of nocturnal pain crises. EVA was estimated $<3$ for both day and night crisis. Total daily tapentadol dose was $300 \mathrm{mg}$.

\subsection{5. $5^{\text {th }}$ and Consequent Medical Visits after 9 Months and after 1 Year of the beginning}

The pain was greatly improved $(\mathrm{EVA}<3)$ and treatment was well tolerated except low constipation.

\section{Discussion}

The use of potent opioid analgesics to treat NP is a controversial area because of concerns about dependence, tolerance, addiction and the general belief that these drugs cannot improve this type of pain. This fact has prompted the creation a term defined as "opiophobia" either by the medical professionals or by the patients. A recent Cochrane review [2] considered the short-term studies lasting less than a day and intermediate-term trials with duration of several days to 12 weeks. The 31 studies included 1237 patients with NP. The short-term studies produced conflicting results, and just over half $50 \%$ indicated that opioids could be better than placebo. Although all intermediate term studies showed that opioids were better than placebo, most studies were small in number of patients and short duration, and methods used which do not cause bias were not widespread. It is likely that all these features lead to the effects of these drugs seem better in clinical trials than they are in clinical practice. It was not possible to define if opioids have advantages over placebo in treating NP over time. Finally, side effects such as constipation, nausea, dizziness and drowsiness were common but were not life threatening. The conclusion was that more controlled clinical trials are needed to determine long-term efficacy, safety and effect on quality of life in these patients.

Oxaliplatin (ACT-078) is a derivative of platinum [3], which is distinguished by its structural configuration and potential damage to DNA tumor's cells. Oxaliplatin have demonstrated effectiveness in various malignancies, especially gastrointestinal tumors. The pathophysiology of neuropathy mediated by this agent is based on an alteration of the voltage-dependent sodium channels, which results in a depolarization process. Other studies have also linked oxaliplatin to induce neuropathy with glutathione deficiency and mitochondrial dysfunction possibly mediated by conformational changes in ATP-dependent potassium channels. The main neurological side effects found after oxaliplatin treatment are sensory neurotoxicity, which can be very limiting for the patient. Electrophysiology studies routinely show ganglionopathy clinically expressed in two forms: either acute or chronic one. First one is the most common, occurring in up to $95 \%$ of patients. It appears during 
infusion of oxaliplatin or few hours after finishing their administration. It's often triggered or aggravated by exposure to cold, self-limited and with a rapid recovery within hours or days. The patient usually refers paresthesia and dysesthesia in the distal portion of the extremities and perioral region, pharyngo-laryngeal dysesthesia ( $1 \%$ to $2 \%$ of cases), neuromyotony and myopathy. Recovery may be complete after the treatment. This fact does not occur with chronic neuropathy presentation, in which the recovery is longer and often incomplete. The chronic form appears when higher cumulative dose of oxaliplatin above $300 \mathrm{mg} / \mathrm{m}^{2}$ are used. Usually, the patient shows symptoms very similar to those described above for this drug neurotoxicity. It has not been proven effective preventive treatment. Methods to treat or prevent oxaliplatin-induced neurotoxicity center around two main concepts: dosing strategies and neuromodulator agents, in particular, intravenous calcium and magnesium infusion, but educating the patient and caregiver is of utmost importance [4].

Tapentadol [5], is a novel opioid analgesic drug dual centrally acting agonist at the mu opioid receptor (MOR) and inhibitor of CNS norepinephrine reuptake (NRI) whose potency is between morphine and tramadol. The Food and Drug Administration (FDA) has approved it for the treatment of moderate to severe acute pain in the form of immediate release tablets and also for chronic pain in its extended release form (PR), last one with a half-life of 12 hours. Its oral bioavailability is estimated around $32 \%$. It is widely distributed, binds $20 \%$ to proteins, it has a $97 \%$ hepatic metabolism and finally it's excreted in the urine by $99 \%$ mainly as conjugated metabolites. These metabolites should have analgesic action but biotransformation of tapentadol by metabolic enzymes results in inactivation. Its effectiveness has been proven in degenerative articular, cancer and diabetic neuropathic pain with a maximum recommended dose of 500 $\mathrm{mg} /$ day. Tapentadol PR (100-250 mg twice daily) was efficacious and provided efficacy that was similar to oxycodone $\mathrm{HCl}$ controlled release (20-50 mg twice daily) for the management of chronic osteoarthritis knee and low back pain, with a superior gastrointestinal tolerability profile and fewer treatment discontinuations [6]. Tapentadol presents an analgesic efficacy comparable to that of strong opioids such as oxycodone and morphine. Its high efficacy has been demonstrated in a range of animal models of acute and chronic, nociceptive, inflammatory, and neuropathic pain as well as in clinical studies with moderate to severe pain arising from a number of different etiologies. At the same time, a favorable gastrointestinal tolerability has been demonstrated in rodents and humans, and advantages over morphine regarding tolerance development and physical dependence were shown in animal studies. Furthermore, a low level of abuse and diversion is beginning to emerge from first post-marketing data. Both mechanisms of tapentadol clinical action have been shown to contribute to the analgesic activity of this drug and to produce analgesia in a synergistic manner, such that relatively moderate activity at the two target sites is sufficient to produce strong analgesic effects [7]. The broad analgesic efficacy of tapentadol PR may simplify chronic pain management by allowing for the treatment of different types of pain with a single analgesic. In addition, tapentadol is associated with a low risk of pharmacokinetic interactions, which permits its use in patients taking multiple medications. Furthermore, the favorable tolerability profile of tapentadol PR may result in improved patient compliance and allow for easy titration and rotation from previous strong opioids [8]. It has been suggested that tapentadol is the first representative of a proposed new class of analgesics, (MOR and NRI) and due to this pharmacological profile, tapentadol can be a good option for the treatment of NP $[7,9,10]$.

This case-report presents the evidence that has led to this suggestion, and outlines how the pharmacology of tapentadol can explain its broad analgesic activity profile and high analgesic potency as well as its favorable tolerability.

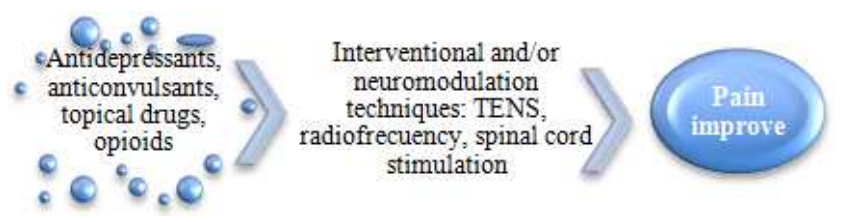

Figure 1.Different approaches for the management of neuropathic pain

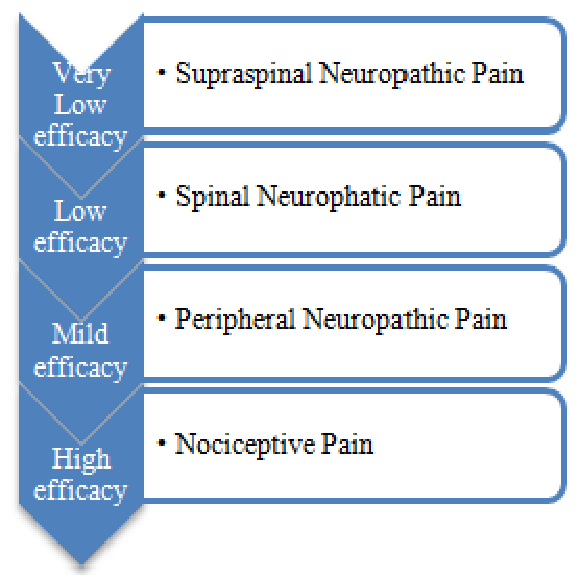

Figure 2. Relative overall opioid responsiveness of different categories of pain

Treatment for NP should be multimodal and $1^{\text {st }}$ line drugs are tricyclic antidepressants (amitriptyline) inhibitors or serotonin reuptake inhibitors (venlafaxine, duloxetine), anticonvulsants (gabapentin and pregabalin) and topical lidocaine or capsaicin for localized painful processes. Moreover, $2^{\text {nd }}$ step opioids like tramadol have been effective as adjuvants. Further, $3^{\text {rd }}$ major opioids (morphine, oxycodone, fentanyl, methadone, buprenorphine, tapentadol) are ordered when the intensity of the pain is greater or prior therapies, including interventional ones, have failed or are contraindicated, although it is postulated that total required dose are higher than those used for inflammatory and/or nociceptive pain [10] (see figure $n^{\circ} 1$ ). These drugs are also effective during the implementation of an analgesic regimen, for breakthrough pain crises and for patients with mixed pain (inflammatory / neuropathic) such as sciatica or cancer pain $[1,8,9,10]$. It has also been estimated a greater responsiveness 
to opioid drugs for peripheral neuropathic pain rather than central one when the injury is originated in the central nervous system (spinal cord and / or brain) [10] (see figure $\mathrm{n}^{\circ} 2$ ). This is fulfilled in our case and tapentadol at doses of $300 \mathrm{mg} /$ day has been shown effective in association with pregabalin 450 $\mathrm{mg} /$ day for control of neuropathic pain caused by severe distal sensory polyneuropathy due to chemotherapy treatment with oxaliplatin.

\section{Conclusions}

The main goal of treatment of NP is pain relief. However, even the most appropriate therapeutic strategy sometimes is only able to reduce pain to a more tolerable level. Despite the variety of available agents and the considerable increase in the number of clinical trials in recent years, medical treatment of neuropathic pain is still considered far from satisfactory. Indeed, less than half of the patients can obtain clear benefit [1].

Clinicians have widely used many different types of drugs for the treatment of NP and also interventional or neuromodulator techniques (TENS, nerve blocks, lesion by conventional or pulsed radiofrequency, spinal cord stimulation) with varying results depending on the source of pain. No drug works on all types and stages of NP, and taking into account the diversity of pain mechanisms and responses of patients, treatment should be individualized and multimodal, including psychological therapy $[1,2]$. In addition to analgesia, it's an important fact to consider other factors such as comorbidities, tolerability, risk of adverse effects, and additional benefits such as sleeping quality, mood or quality of life, and cost / effectiveness for either the patient or Health System [7,8,9].

The treatment of oxaliplatin-induced neurotoxicity needs to start at the bedside. Nursing staff and oncologists should inform patients to avoid contact with cold objects, food, and beverages. This can prevent paresthesias and more importantly pseudo laryngospasm. Promising strategies exist that include dosing modification and neuromodulatory agents, although larger trials need to be conducted to confirm efficacy [4].

Antidepressants and antiepileptic drugs have demonstrated the best evidence-based efficacy for coping with NP. Tapentadol due to its dual mechanism of action can be a drug highly effective and very well tolerated in severe neuropathic pain, as demonstrated in this polyneuropathy clinic case induced by oxaliplatin. Tapentadol is destined to become a $1 \mathrm{st}$ line opioid therapy within the multidisciplinary management of these patients $[2,5,7,8,9,10]$.

\section{Acknowledgements}

Dr. Borja Mugabure would like to thank all the medical and nursery members from the Chronic Pain Unit, Donostia University Hospital, for their support.

\section{References}

[1] Bouhassira D, Lantéri-Minet M, Attal N, Laurent B, Touboul C.Prevalence of chronic pain with neuropathic characteristics in the general population. Pain. 2008; 136(3): 380-7.

[2] McNicol ED, Midbari A, Eisenberg E. Opioids for neuropathic pain. Cochrane Database Syst Rev. 2013; 8: CD006146.

[3] Velasco R, Bruna J. Chemotherapy-induced neuropathy: a problem unresolved. Neurology. 2010; 25 (2): 116-131

[4] Saif MW, Reardon J. Management of oxaliplatin-induced peripheral neuropathy. Ther Clin Risk Manag 2005; 1(4): 249-258

[5] Afilalo M, Morlion B. Efficacy of tapentadol ER for managing moderate to severe chronic pain. Pain Physician. 2013; 16 (1): 27-40.

[6] Lange B, Kuperwasser B, Okamoto A, Steup A, Häufel T, Ashworth J, et al. Efficacy and safety of tapentadol extended release for chronic osteoarthritis pain and low back pain. Adv Ther. 2010; 27 (6): 381-99.

[7] Tzschentke TM, Christoph T, Kögel BY. The mu-opioid receptor agonist/noradrenaline reuptake inhibition (MOR-NRI) concept in analgesia: the case of tapentadol. CNS Drugs 2014; 28 (4): 319-29

[8] Sánchez Del Águila MJ, Schenk M, Kern KU, Drost T, Steigerwald I. Practical Considerations for the Use of Tapentadol Prolonged Release for the Management of Severe Chronic Pain. Clin Ther. 2014; S0149-2918 (14) 00445-7. [Epub ahead of print]

[9] Pierce DM, Shipstone E. Pharmacology: tapentadol for neuropathic pain. Am J Hosp Palliat Care. 2012; 29 (8): 663-6

[10] Smith HS. Opioids and neuropathic Pain. Pain Physician. 2012; 15 (3 Suppl): ES 93-110 